\title{
Nocardia asteroides EN ABSCESO PULMONAR INTERPRETADO COMO DE ORIGEN TUBERCULOSO: CASO FATAL.
}

\author{
(Nocardia asteroides in a pulmonary abscess considered to be of a \\ tuberculous origin: Fatal case.)
}

\author{
R. Salim*, C. Villagra de Trejo** y R. Runco de Laborda* \\ * Cátcdra de Micología, Instituto de Microbiología "Dr. Luis C. Verna", \\ Facultad de Bioquímica, Química y Farmacia, Universidad Nacional de Tucumán, \\ Ayacucho 491 - (40)(0) San Miguel de Tucumán. \\ ** Laboratorio de Bactcriología, Hospital del Niño Jesús, \\ Pje. Hungría 700) - (4000) San Migucl de Tucumán.-
}

Palabras clave: $N$. asteroides, absceso pulmonar, tuberculosis

Key Words: $N$. asteroides, pulmonary abscess, tuberculosis

\section{RESUMEN}

Se informa el hallazgo de cultivos puros de $N$. asteroides a partir cle la colección purulenta obtenicla por drenaje quirírgico de un absceso en base pulmonar derecha, de $6 \mathrm{~cm}$. de diámetro, en una niña de 13 años de edad con desnutrición de segundo grado $y$ antecedentes de tuberculosis.

Lo prolongado de la evolución diagnóstica, el cuadro infeccioso inespecifico y el probable deterioro inmunolígico pudieron haber producido cierta demora en el diagnóstico y por lo tanto asociarse para el desenlace fatal.

\section{INTRODUCCION}

Las cspecies de Nocardia han sido reconocidas como patógenas oportunistas en pacientes inmunocomprometidos, con una amplia variedad de condiciones predisponentes: tuberculosis, SIDA, cirrosis, Icuccmias, linfomas, enfermedades pulmonares crónicas, transplantes de órganos y en pacientes que reciben tratamientos inmunosupresores $(5,7,11,16)$.

A partir de las partículas de polvo, las formas vegetativas de Nocardia inhaladas, son rápidamente fagocitadas pero no destruídas por los fagocitos, por lo que pueden desarrollarse en su intcrior $(3,4,9)$. Si la respuesta del hospedador no es suficiente para detener su crecimiento intracelular, se desarrolla una infección progresiva que

\section{SUMMARY}

The present paper reports the isolation in pure cultures of $N$. asteroides from the purulent content obtained from the surgical drainage of a $\sigma \mathrm{cm}$ diameter abscess in the right lower pulmonary lobe, of a thirteen year old girl with a grade II malnutrition and a clinical history of tuberculosis.

The extent of the diagnostical evolution, the probable immunological damage and nonspecific clinical features may have produced certain delav in the diagnosis and therefore be associated with the fatal outcome.

pucde mani festarse como una neumonía que se expande o circunscribe en un absceso pulmonar, dando un cuadro clínico y patológico variable y no específico $(4,11)$. Pudiendo permanecer localizada o diseminarse por vía hematógena, virtualmente a todos los órganos $(1.5,6,7$, $8,11,12)$.

A menudo, simula otras enfermedades como tuberculosis, enfermedades piógenas, tumores y una varicdad de micosis por lo que es facilmente mal diagnosticada ( $t$, $5,7,11$ ).

El compromiso pulmonar está asociado con una variedad de presentaciones radiográficas, incluyendo consolidación uni o bilatcral, infiltrados intersticiales difusos, Icsiones en masa solitaria, cavitación y derrame pleural $(2,4.1,16)$.

El objctivo del prescnte trabajo fue informar el 
hallazgo de cultivos puros de $N$. asteroides como agentc de infección oportunista en una niña de 13 años de cdad con absceso pulmonar .

\section{CASO CLINICO}

Paciente de sexo femenino de 13 años de cdad, nacida en la provincia de Tucumán (R. Argcntina). Consulta en el Hospital de Concepción por síndrome febril prolongado, tos, disnea y dolor en hemitórax derecho. Sc diagnostica como neumonitis incspecífica y es medicada empíricamente durante sicte dias. Las muestras de lavado bronquial fucron negatiras para micobacterias. La reacción de PPD resultó negativa. Los cxamenes de laboratorio revelaron una critrosedimentación de $97 \mathrm{~mm} \mathrm{cn}$ la la. hora y 13.0(0) leucocitos/mm3. La radiografia de tórax muestraron imágenes algodonosas difusas $\mathrm{cn}$ ambas bascs pulmonarcs. por lo que se deriró al Hospital del Niño Jcsús para interconsulta con el Servicio de Ncumonología.

Sus antecedentes clínicos comenzaron a los 10 años de cdad, cuando se le diagnostica cn Ncuquén (R. Argentina) una tuberculosis pulmonar. realizandose dos años de tratamicnto cspecífico con Rifampicina y Nicotibina.

$\mathrm{Al}$ ingreso. pudo apreciarse una paciente levemente cnferma. sobre un fondo crónico de desnut rición de segundo grado (déficit del $32 \%$ ). febril, con palide culanıenumeosa moderada. uñas en vidrio de reloj. hábito torácico. espiración prolongada con leve disminución de la cntrada de aire en base derecha y el resto del cxamen físico sin particularidades.

Los datos de laboratorio mucstran una critrosedimentación de $87 \mathrm{~mm}$ la 1ra. hora y una leucocitosis de $18.500 / \mathrm{mm}^{3}$.

La búsqueda de micobacterias es negativa en el lavado bronquial, aislándose $P$ seudomonas aeruginosa. Los hemocultivos son negativos.

Es medicada con Rifampicina. Ciprofloxacina y Fluconazol. La tomografía axial computarizada (TAC). permite reconocer una imágen hipodensa heterogénea de contornos densos y zonas hipodensas múlliples sobre la trama pulmonar. que corresponden a enfisemas locilizados.

Evolucionó con febrículas y con puntada de costado. La leucocitosis ascendió a $62.6(6)\left(\mathrm{mmm}^{3}\right.$. Unil nueva radiografía de tórax mostró en base derecha. una imágen radiopaca redondcada con zonas radiolúcidas en su interior. Sc inicia tratamicnto con Cefuroxina.

A los 45 días desde su ingreso, el lavado bronquial es negativo para micobacterias y hongos: se aislan abundantes colonias de cocobacilos Gram (-) y diplococos Gram (+) sin identificar.

Se rota antibióticos a Cefotavina y Rifampicina.

El cuadro persiste sin variantes y con compromiso del estado general. En ese momento los ivámenes de laboratorio mostraron una leucocitosis de $1 .+.(0) 00 / \mathrm{mm}^{3}$ y una acentuada aceleración de la critrosedimcitación: 11 () mm la la. hora.

La ecografia torácica confirma en el hemitórax derecho una imagen redondeada de $4+\times 60 \mathrm{~mm}$ do diámetro, de límites densos y netos. con contenido líquido. que puede corresponder a un absceso.

La TAC, muestra una imagen de consolidación vinculada a compicma basal posterior derecho.

A los 55 dias desde su ingreso se decide realizar toracolomia en hemitórax derecho. En cl lóbulo inferior se constitan grandes cavitaciones con contenido de tipo cascoso. Además se observa cavilación en diafragma. Sc cmvia material para citologia y cullivos. Se agrega Isoniazida y Pirazinamida.

El laboratorio de Micologia informa la observación de filamentos Gram (+) parcialmente ácido-rcsistentes en los cxámenes microscópicos coloreados de la colección purulcnta. compatibles con Nocardia. Se retira el tratamicnto tuberculostático y sc agrega TrimetroprimalSulfametoxizol (3) $\mathrm{mg} / \mathrm{kg} /$ día).

La paciente presenta cianosis distal. palidez cutanocomucosa. ficbre y mal estado gencral. La radiografia de tórax mucstra opacidad en aumento en hemitórax irquicrdo y mayor recexpansión del derecho.

El informe anatomopatológico indica imagen sugerente de tuberculosisavascular en severa desnutrición. diagnosticandose sin cmbargo bronconeumonía histioide con necrosis secundaria.

Con crolución tórpida ý declinación de los parámetros vitales durante quince días, la paciente fallece por paro respiratorio y cardíaco.

El laboratorio de Micología confirma el desarrollo de cultivos positivos de Nocardia asteroides.

No se efectuó nccropsia.

\section{ESTUDIO MIICROBIOLOGICO}

A partir del licquido obtcnido por drenaje quirúrgico del absceso se realizaron exicndidos coloreados con Gram y Kinyoun. lo que permitió la visualización de filamentos bacterianos ramificados, fragmentados y cuerpos cocobacilares Gram (+) parcialmente ácido-resistcntes a la decoloración con $\mathrm{H} 2 \mathrm{SO}+$ aq. $0.8 \%$ compatibles con Nocurdia.

Siguicndo nuestra técnica de aislamicnto como se describe en trabajos previos (I0. 18). el matcrial fuc sembrado por quintuplicado en el medio de Czapeck líquido sin sacarosa con anzuclo parafinado estéril. Los tubos fucron incubados a $37^{\circ} \mathrm{C}$. A partir de los $1+$ dias comenyó a observarse un desarrollo anaranjacto sobre el anzuclo parafinado, que fuc traspasado a Sabouraud agar. 
Agar gliccrinado 5\% y agar $\mathrm{BHI}$. manteniendose la misma $\mathfrak{l}^{\circ}$ de incubación. A los pocos dias. cn todos los medios sembrados comenzó cl desarrollo de colonias glabras. plegadas. de color anaranjado pálido. El cxamen microscopico de estas colonias mostró filamentos finos. ramificados y fragmentados en elementos bacilares y cocoides Gram (+), parcialmente ácido-resistentes.

Para su identificación se realizaron estudios morfológicos y fisiológicos (10. 18) que dicron los siguientes resultados: producción de urcasa (+); hidrólisis de gelatina y almidón (-): descomposición de xantinia. hiposantina. tirosina y cascína (-). Hubo desarrollo en gelatina $(0.4 \%$ con acide\% del pH, no hubo producción de ácido a partir de: arabinosa, inositol, lactosa, maltosa, mclibiosa. ribitol. D-xilosa y D-galactosa.

La interpretación de estas prucbas permitió su identificación como $N$. asteroides.

\section{DISCUSION}

Desde el primer caso documentado de nocardiosis en 18\%). las infecciones por especies de Nocardia han sido reconocidas como una scria infección humana cn aumento, que puede ser causa significatira de morbimorlalidad ( $t$ ).

Las especies de Nocurdia son los principales agentes de micetomas humanos en Tucumán.. Su aislamiento de suclos de distinas áreas nos permitió establecer su anplia distribución en la provincia (18). El $68 \%$ de las especies aisladas resultaron experimentalmente patógenas (19).

Quizás en nuestro medio. la baja incidencia de nocardiosis se debe a la falta de reconocimiento de la infección.

El desarrollo de cultivos puros de $N$. asteroides en todos los tubos, sembradosa partir de la colección purulenta del absceso. nos lleva a pensar que podría tratarse de un caso de nocardiosis oportunista asociado a tuberculosis.

Sc ha estimado que el 5\% de todos los pacientes con tubcrculosis. tambićn desarrollan nocardiosis y hay varios informes de infecciones mixlas a Nocardia y Mycobacterium tuberculosis. Las razoncs para csta combinación aún son desconocidas $(+.15,16)$. En un estudio reciente (10). hemos detcelado un 7.6\% de cultivos positivos de Nocurdia cn pacicntes con compromiso pulmonar crónico. cn mucstras cnviadas únicamente para análisis bacıcriológico de rutina. Resulta llamativa la preralencia de $N$. asteroides en pacientes con tuberculosis (75\%).

Nuestra paciente presentó como rayones potenciales de inmunocompromiso. no sólo sus antecedentes de tuberculosis y la antibioticotcrapia prolongada, sino tambićn su desnutrición de scgundo grado.

El caso que describimos resulta llamativo además por la edad de la paciente ya que la afección es más frecucnte cll cl varón adulto mayor de 40 años que en la mujer (t).

Como las manifestaciones clínicas, radiográficas o histopatológicas de nocardiosis no son específicas, su diagnóstico cs difícil si no sc recurre a cstudios cspecíficos de laboratorio ( $11,13,14)$.

El desalío. es descubrir la infección lo más tempranamente posible. Por cllo considcramos de gran valor cl diagnóstico presuntivo rápido basado en la demostración del microorganismo en los cxamenes coloreados. para permitir al clínico comenzar un tratamiento tentativo hasta que cl laboratorio pucda confirmar cl diagnóstico.

Las especics de Nocardia requicren condiciones especiales de cultivo para su aislamicnto. no realizados de rutina en los laboratorios de bactcriologia (16). El aislamicnto de cultivos puros de Nocardia a partir de materiaIcs densamente contaminados con bacterias. tales como csputo y lavado bronquial. es una de las mayores dificultades. Las bactcrias acompañantes, en especial las de crecimicnto rápido, pucden opacar pequeños números de colonias de Nocardia cuyo crecimiento es más lento (2-4 scinanas). Existe cntonces el peligro de que los cultivos scanl cquivocadamente descartados antes de que pase el tiempo suficiente para su desarrollo. Este hecho, sumado al largo ticmpo en su postcrior identificación, son los mayores obstáculos para la detccción de la infección (10, 17).

En el caso que nos ocupa. es probable que los cultivos de lavado bronquial resultaron negativos al ser procesados siguiendo la técnica de rutina en bactcriología de tuberculosis. La homogenización y los procesos de descontaminación de las muestras clínicas, pucden matar a estos agentes $(13,15,17)$. Además la pacicnte recibió tratamiento antituberculoso cuando se procesaban las muestras.

En nucstros trabajos previos encontramos que el mélodo del anzuclo parafinado. de rutina en los laboratorios de Micología, es el procedimiento más eficaz en comparación a los otros métodos de aislamicnto de Nocardia desde la microbiota comensal, ya que cstas últimas son incapaces de utilizar la parafina como única fucnte de C.

Las nocardiosis. son dificiles de tratar y la mayoría de los antimicrobianos comunmente usados para el tratamiento de los procesos neumónicos infecciosos, son incficaces. Los ćxitos tcrapćuticos han aumentado durante los últimos 10 años. quizás debido a una pronta administración de ćstos. que incluyen. no sólo a TrimetroprimaSulfamctoxazol. sino tambićn a Ciprofloxacina, Cefalotina, Cefolaxina. Amicacina e Imipencm. Aún así, un número significativo de pacientes fallecen, subrayando la necesidad de antimicrobianos de nueva generación junto a métodos diagnósticos más rápidos. El tratamiento 
prolongado cs indispensable para controlar la nocardiosis (t. 11).

Estas consideraciones nos llevan a las siguientes conclusiones:

- Es importante mantener un alto grado de sospecha clíniča de nocardiosis en cualquicr proceso neumónico infeccioso en pacientes inmunocomprometidos. Quizás la baja incidencia de nocardiosis se debe a la falta de reconocimiento de la infección.

- La detección de Nocardia debiera incluírse en cl diagnóstico diferencial en pacientes cuyos cultivos iniciales son negativos.

- Promover un cambio de actitud en cl laboratorio a fin de que se incorpore de rutina la búsqueda de Nocardia.

-Enfatizar el valor de la demostración de los microorganismos en los examenes microscópicos que permitan comenzar una terapia tentativa temprana.

- Implementar el método del anzuclo parafinado en las muestras clínicas sospechosas, critando la digestión y la descontaminación previa.

- Destacar la necesidad de mćlodos diagnósticos más rapidos.

- Considerar sistemáticamente a las Micosis y a las Nocardiosis como parte del diagnóstico diferencial de la tubcrculosis.

\section{REFERENCIAS}

1. Almekinders, L. \& Lachiewic\%, P.(1989). Nocardia osteonyelitis Case report and review of the literature. Orthopedies 12:1583-85

2. Balikian, J.; Ilerman. P. \& Kopit, S. (197\&). Pulmonary nocardiosis. Radiology 126:569-573

3. Beaman, B. L. \& Beaman, L. (1994). Nocardia species host-parasite relationships. Clin. Microbiol. Rev. 7:213-264

4. Beaman, B. L. (1993). Nocardial infections. In: J.W. Murphy et. al. (Eds.) Fungal Infections and Immune Responses. Plenum press. N.Y. 24: $53.3-554$

5. Boiron, P.F.; Provost, F.; Cheveier, G \& Dupont, 13. (1992). Review on nocardial infections in France 1987-1990. Eur. J. Clin. Microbiol. Infect. 1)is. 11:709-714

6. Borghi, A.; Mujica, M, \& Ponesa, A. (1992). Nocardiosis cerehral debidat a $N$. asteroides. Rev. Arg. Micologia 15:3-6

7. Cherubin, S.E. (1992). Nocardiosis in patients with AIDS. Clin. Inlect. Dis. $15: 370)-377$

8. Douglas, R. M.; Grove, D.; Elliot,F.; Looke, I). \& Jordan, A. (1991). Comcal ulceration due to $N$. asteronides Aust. N.Z.... Ophtalmol 19:317320

9. Filice, G.A.; Beaman, B.L.; Krick, J. \& Remington,F. (1980). l:flects of human neutrophils and monocytes on N. asteroides: Failure of killing despite occurrence of the oxidative metaholic hurst. J. Infect. Dis. 1+2: $4.32-+38$

10. I aborda, R.R. \& Salim, R. (1995). Detecion de especies de Nocurdia aisladas de pacientes con compromiso pulmonar cronico. Boletin Mieolígico 10:33-36
11. MeNeil, M. \& Brown,J. (1994). The medically important aerobic Actinomycetes: Epidemiology and Microbiology. Clin. Microbiol. Rev. 7:357-417

12. Monacci, M. G. \& Pons de Storni,L. (1984). Nocardiosis pulmonar. Descripción de un caso. Rev. Arg. de Micologia 7:24-25

13. MIurray, P.; Ilecren. R. \& Niles,A. (1987). Eflect of decontamination procedures on recovery of Nocardia species. J. Clin. Microbiol. 25:2010 2011

14. Negroni, R.; Masini, R.; García, O. \& Malliolo, E. (1991). Nocardiosis sistemica por $N$. caviae con signo-sintomatologia preponderantemente hepítica. Rev.Arg. Micologia 14:24-25

15. Neismeister, R.; Patterson,N.; Cocklin, J. \& IIarada,G (1971). The isolation of pathogenic fungi from sodium hydroxide-processed sputum specimens from patients suspected to have tuberculosis. Am. J. Clin. Pathol. $54: 201-203$

16. Simpsen, G.,; Stinson, E.; Eggert, M. \& Remington, J. (1981). Nocardial infections in the in the immunocompromised host: a detailed study in a delined population. Clin. Infect. Dis. 18:348-353

17. Stevens, D. (1983). Clinieal and clinical laboratory aspects of nocardial infection. J. Hyg. 91:377-384

18. van Gelderen de Komaid, A.; de Laborda, R.R. \& Salim,R. (1987). The natural occurence of Nocurdia in soil of Tucuman: Physiological characteristics Mycopath. 99:15-19

19. experimental pathogenicity in Swiss mice of various strains of Nocardia isolated in soil of Tucumain (Argentina). Mycopath. 98:9-12 